\title{
Física e música em consonância
}

\author{
(Physics and music in consonance)
}

\author{
Mario Gotd 1 \\ Departamento de Física, Centro de Ciências Exatas, Universidade Estadual de Londrina, Londrina, PR, Brasil \\ Recebido em 15/10/2008; Revisado em 11/2/2009; Aceito em 18/2/2009; Publicado em 26/6/2009
}

São examinadas as condições físicas e matemáticas da consonância das ondas sonoras, estabelecendo-se uma relação entre suas frequências fundamentais. Mostra-se que é independente de fases e amplitudes relativas além de ser válida para todas as suas componentes harmônicas, concluindo-se que esta relação de consonância assim definida depende apenas das frequências fundamentais. Por fim, examina-se como esta relação se manifesta na estrutura da escala musical.

Palavras-chave: física e música, relação de consonância, escala musical.

Physical and mathematical conditions of consonance of sound waves are examined and a relation between its fundamental frequencies is established. It is shown that it is independent from the relative phases and amplitudes as well as the validity for all the harmonic frequencies. At last, it is discussed how this consonance relation manifests itself in musical scales.

Keywords: physics and music, consonance relations, musical scales.

\section{Introdução}

A música é a arte dos sons e a consonância das ondas sonoras é o que torna possível a música na nossa vida. As regras para se combinar sons consonantes são bem conhecidas, tendo sido estabelecidas ao longo da evolução da música. Os elementos básicos são as notas e os intervalos entre as notas, cujas propriedades principais são a frequência (da nota) e a consonância (do intervalo). Se duas notas musicais tem frequências $f_{1}$ e $f_{2}$, respectivamente, o intervalo entre estas notas é definido pela relação $r=f_{2}: f_{1}$. Embora a frequência seja uma grandeza contínua, a música é composta por sons consonantes, sendo que os intervalos de interesse musical se manifestam como frações de uma oitava, assim chamada por conter oito notas (dó, ré, mi, fá, sol, lá, si, Dó) dentro do intervalo de frequência $f(D o ́): f(d o ́)=2$. Estas oito notas definem a escala musical básica conhecida como a escala de dó maior.

Em relação à nomenclatura [1], as denominações das notas musicais estão relacionados com a língua dominante dos países, principalmente, conforme mostra a
Tabela 1. Países de idiomas diferentes tendem a adotar a nomenclatura inglesa devido ao predomínio do inglês como linguagem universal. Neste texto os nomes das notas, exceto os da oitava central, serão em letras minúsculas, os acentos podendo ser omitidos. Quanto às oitavas, serão indicadas por índices inferiores variando de 0 a 8.

A chamada música ocidental é baseada na escala de entonação justa, um conjunto de notas musicais no intervalo de frequências de $f_{0}$ a $f_{1}=2 f_{0}$ que define uma oitava, $f_{0}$ uma frequência de referência. A audição humana é sensível a frequências entre $20 \mathrm{~Hz}$ a $20.000 \mathrm{~Hz}$, e um piano típico cobre 7 oitavas, das notas $l a_{0}$ a $d o_{8}$, com frequências de $27,5 \mathrm{~Hz}$ e $4.224 \mathrm{~Hz}$, respectivamente, tendo como padrão de afinação a nota $l a_{4}$ (quarta oitava, central) com frequência atribuída de $440 \mathrm{~Hz}[2$, 3]. A Fig. 1 mostra o teclado do piano; a nota dó central, de referência, próxima à chave, é o do 4 . É claro que o teclado do piano contém muito mais notas do que as oito (por oitava) necessárias para a escala básica de dó maior. Esta questão será retomada adiante ao tratar das construções das escalas musicais.

Tabela 1 - Denominações das notas musicais estão relacionados com a língua dominante dos países.

\begin{tabular}{lccccccc}
\hline Línguas latinas em geral & Dó & Ré & Mi & Fá & Sol & Lá & Si \\
\hline Francês & ut & rè & mi & fà & sol & là & si \\
\hline Inglês e alemão & C & D & E & F & G & A & B \\
\hline
\end{tabular}

\footnotetext{
${ }^{1}$ E-mail: mgoto@uel.br. 


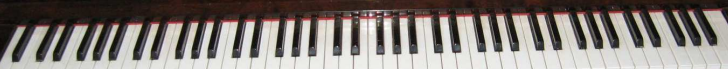

Figura 1 - Teclado típico de um piano.

\subsection{Escala pitagórica}

A origem da escala musical remonta ao matemático grego Pitágoras que, usando um monocórdio com um suporte móvel entre as extremidades fixas da corda vibrante, identificou as relações entre as frequências como os fatores preponderantes para a consonância dos sons. A Fig. 2 ilustra um monocórdio, o suporte móvel dividindo a corda tensionada em duas partes de comprimentos $L_{1}$ e $L_{2}$. Pitágoras percebeu que os sons produzidos pelas partes com relações de comprimentos $L_{1} / L_{2}$ iguais a $2 / 1,3 / 2$ e $4 / 3$ eram particularmente agradáveis enquanto que quaisquer outras combinações arbitrárias resultavam desagradáveis. Dois sons resultando numa combinação agradável são ditos consonantes, de outro modo são dissonantes.

Uma corda de comprimento $L$ tensionada e fixa nas duas extremidades tem modos de vibração definidos por comprimentos de onda $\lambda_{n}$ satisfazendo [4]

$$
\lambda_{n}=\frac{2 L}{n},
$$

para $n$ assumindo valores inteiros $1,2,3, \ldots$, etc. Comprimento de onda e frequência se relacionam com a velocidade de propagação da onda sobre a corda, $v=f_{n} \times \lambda_{n}$, de modo que

$$
f_{n}=\frac{v}{\lambda_{n}}=\frac{n v}{2 L} .
$$

Considerando apenas o modo fundamental $(n=1)$, as relações de frequência ficam $f_{2} / f_{1}=L_{1} / L_{2}$. Em notação atual, as relações acima definem as notas $d o_{1}$, $\mathrm{fa}$, sol e $\mathrm{do}_{2}$ com a relação de frequência $3 / 2: 4 / 3=$ $9 / 8$ entre as notas $f a$ e sol, que define o intervalo de um tom. A relação 2/1 corresponde ao intervalo de uma oitava, neste caso de $d o_{1}$ a $d o_{2}$.

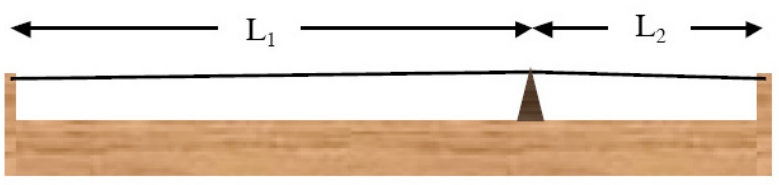

Figura 2 - O monocórdio (ou monocorda), corda tensionada e fixa nas duas extremidades, com um suporte móvel que permite variar o comprimento da corda.

Tomando como referência a nota $d o_{1}$ definem-se as notas re um tom acima e mi outro tom acima do re. Com o mesmo procedimento, tendo como base a nota sol, definem-se as notas la e si, completando a escala de Pitágoras, na talela 2. A Tabela 3 mostra os intervalos entre as notas adjacentes numa oitava, de $d o_{1}$ a $d o_{2}$. Os intervalos entre as notas $m i-f a$ e $s i-d o_{2}$ resultam 256/243, intervalo que define o meio tom ou semitom.

Por um vício de linguagem é comum dizer que semitom é a metado do tom - assim seria se o intervalo fosse a diferença das frequências. O intervalo é a razão entre as frequências e, a rigor, espera-se que o intervalo de um tom contenha dois intervalos de semitons. Significa que se $r$ for o intervalo de semitom, $r \times r=r^{2}$ deve corresponder ao intervalo de um tom. Na escala pitagórica isto é satisfeito de forma aproximada, pois

$$
r^{2}=\left(\frac{256}{243}\right)^{2} \simeq 1,1099
$$

e o intervalo de um tom é $9 / 8=1,125$. Significa, por exemplo, que meio tom acima de do e meio tom abaixo de re não são equivalentes.

Tabela 2 - Notas e os respectivos intervalos em relação à primeira nota $d o_{1}$, na escala pitagórica.

\begin{tabular}{cccccccc}
\hline$d o_{1}$ & $r e$ & $m i$ & $f a$ & sol & la & si & $d o_{2}$ \\
\hline 1 & $9 / 8$ & $81 / 64$ & $4 / 3$ & $3 / 2$ & $27 / 16$ & $243 / 128$ & 2 \\
\hline
\end{tabular}

Tabela 3 - Notas e os intervalos entre duas notas consecutivas, na escala pitagórica.

\begin{tabular}{ccccccc}
\hline$d o_{1}-r e$ & $r e-m i$ & $m i-f a$ & $f a-s o l$ & sol $-l a$ & la - si & si-do \\
\hline $9 / 8$ & $9 / 8$ & $256 / 243$ & $9 / 8$ & $9 / 8$ & $9 / 8$ & $256 / 243$ \\
\hline
\end{tabular}

\subsection{Escala de entonação justa}

Consonância e dissonância não são conceitos absolutos, e o astrônomo grego Ptolomeu adicionou às relações $3: 2$ : 1 de Pitágoras as relações $4: 5: 6$, considerandoas tão consonantes quanto as anteriores. Tendo como referência a nota $d o$, resulta o conjunto de intervalos
$\{1,5 / 4,6 / 4=3 / 2\}$ correspondente ao conjunto das notas $\{d o, m i, s o l\}$. Tomando a nota sol como referência, o mesmo conjunto de intervalos multiplicados por $3 / 2$ fica $\{3 / 2,15 / 8,9 / 4\}$ que define o conjunto das notas $\left\{s o l, s i, r e_{2}\right\}$. Por fim, o mesmo conjunto inicial de intervalos deslocados abaixo de $d_{2}$ resulta no conjunto $\{4 / 3,5 / 3,2\}$ correspondente ao conjunto das 
notas $\left\{f a, l a, d o_{2}\right\}$. O intervalo $9 / 4$ de $r e_{2}$ (no segundo conjunto) fica oitava acima e, dividido por 2 , corresponde ao intervalo $9 / 8$ de $r e$.

Este conjunto de notas com os respectivos intervalos em relação à nota $d o_{1}$, na Tabela 4 , é a base da chamada escala de entonação justa. A Tabela 5 traz os intervalos entre as notas adjacentes, mostrando os intervalos de tom maior $(9 / 8=1,125)$, de tom menor $(10 / 9=1,111 \cdots)$ e de semitom $(16 / 15=1,0666 \cdots)$. $\mathrm{O}$ conjunto das oito notas, de $d o_{1}$ a $d o_{2}$, inclusive, define a escala de dó maior. As escalas musicais se repetem em oitavas, e a Tabela 6 relaciona as frequências correspondentes às notas da oitava central da escala de entonação justa, tendo como padrão a frequência $f(L a ́)=440 \mathrm{~Hz}$.

Tabela 4 - Notas e os respectivos intervalos em relação à primeira nota $d o_{1}$, na escala de entonação justa.

\begin{tabular}{cccccccc}
\hline$d o_{1}$ & $r e$ & $m i$ & $f a$ & sol & la & si & $d o_{2}$ \\
\hline 1 & $9 / 8$ & $5 / 4$ & $4 / 3$ & $3 / 2$ & $5 / 3$ & $15 / 8$ & 2 \\
\hline
\end{tabular}

Tabela 5 - Notas e os intervalos entre duas notas consecutivas, na escala de entonação justa.

\begin{tabular}{ccccccc}
\hline$d o_{1}-r e$ & $r e-m i$ & $m i-f a$ & $f a-s o l$ & sol $-l a$ & la - si & si $-d o_{2}$ \\
\hline $9 / 8$ & $10 / 9$ & $16 / 15$ & $9 / 8$ & $10 / 9$ & $9 / 8$ & $16 / 15$ \\
\hline
\end{tabular}

Tabela 6 - Intervalos em relação a Lá e as frequências (em Hertz) correspondentes às notas da oitava central na escala de entonação justa.

\begin{tabular}{cccccccc}
\hline Dó & Ré & Mi & Fá & Sol & Lá & Si & Dó \\
\hline $3 / 5$ & $27 / 40$ & $3 / 4$ & $4 / 5$ & $9 / 10$ & 1 & $9 / 8$ & $6 / 5$ \\
264 & 297 & 330 & 352 & 396 & 440 & 495 & 528 \\
\hline
\end{tabular}

A primeira nota $(d o)$ é a tônica, a terceira $(m i)$ a mediante e a quinta (sol) a dominante. A tônica dá o nome ou a tonalidade da escala, a primeira delas sendo a de dó maior. O padrão se repete em quintas, definindo mais sete escalas de outras tonalidades em ordem ascendente. Para manter o padrão de intervalos (tom-tom-semitom-tom-tom-semitom), recorre-se a notas alteradas de meio tom para cima (sustenido, símbolo \#) em posições específicas. Por exemplo, a próxima escala é a de sol maior, com o conjunto de notas $\left\{s o l, l a, s i, d o, r e, m i, f a_{\#}\right.$, sol $\}$, a nota $f a$ alterada para $f a_{\#}$. Em seguida vem a escala de re maior contendo as notas alteradas $f a_{\#}$ e $d o_{\#}$, e assim por diante. Em ordem descendente, usando alterações de meio tom para baixo (bemol, símblo $b$ ) define-se mais sete escalas, começando pela de $f a$ maior com a nota alterada $s i_{b}$, depois $s i_{b}$ maior com as notas alteradas $s i_{b}$ e $m i_{b}$, e assim por diante.

Para cada uma das escalas maiores, existe uma menor relativa. A relativa menor de do maior é a $l a$ menor, definido pelo conjunto de notas (sem alterações) $\{l a$, si, do, re, $m i, f a$, sol, la $\}$ com o padrão de intervalos (tom-semitom-tom-semitom-tom-tom). Escalas maior e menor diferem nas posições relativas dos intervalos de tom e semitom, estas diferenças produzindo sonoridades que expressam sentimentos antagônicos, alegres (escalas maiores) ou tristes (escalas menores).

Quanto mais notas mutuamente consonantes contiver mais possibilidades oferece a escala e, neste sentido, a escala de entonação justa é mais rica que a de Pitágoras. Esta questão será melhor avaliada na secção 2, que trata das condições de consonância.

Em termos físicos, o som natural pode ser tratado como uma combinação linear de ondas sonoras com frequências harmônicas $f_{n}=n f_{0}$ em relação a uma frequência fundamental $f_{0}$ para $n$ inteiros $(n=$ $1,2,3, \ldots)$. As frequências harmônicas são consideradas consonantes com a fundamental, a composição harmônica sendo uma característica da fonte sonora, e define uma das propriedades do som, o timbre, muitas vezes caracterizado como qualidade do som. O som é produzido pelas vibrações mecânicas de materiais que constituem a fonte sonora contendo, em princípio, todas as frequências harmônicas. Como todo sistema mecânico vibrante real é amortecido, e esse amortecimento depende da frequência e do material, o resultado é a supressão de algumas das componentes harmônicas, moldando assim o timbre.

\subsection{Escala equitemperada}

A escala de entonação justa ou a escala de Pitágoras, conhecidas como escalas naturais, apresentam algumas dificuldades de ordem prática por conter relações de frequência desiguais, o que dificulta a transposição de uma sentença musical ou a execução de uma peça músical usando instrumentos com diferentes formas de afinação. Para contornar estas dificuldades foi criada a escala cromática ou temperada, construída de tal modo que uma oitava contenha exatamente 12 notas separadas em intervalos iguais, de razão $r$,

$$
\begin{aligned}
& f_{0}, f_{1}=r f_{0}, f_{2}=r f_{1}=r^{2} f_{0}, f_{3}=r^{3} f_{0}, \cdots, \\
& f_{12}=r^{12} f_{0}=2 f_{0},
\end{aligned}
$$

onde $f_{0}$ é uma frequência de referência e $f_{12}=r^{12} f_{0}=$ $2 f_{0}$ é a frequência da nota oitava acima, resultando

$$
r={ }^{12} \sqrt{2} \simeq 1,0594631 .
$$

Esta razão $r$ define o intervalo de semitom e $r^{2}$ o intervalo de um tom. A escala de igual temperamento, atualmente, é de uso universal como padrão de afinação 
da maioria dos instrumentos musicais. Instrumentos musicais que tem notas definidas, como o piano, somente são possíveis na escala de igual temperamento, capaz de acomodar todas as escalas usando um número mínimo de notas musicais.

$\mathrm{Na}$ prática a teoria musical utiliza as qualidades sonoras da escala de entonação justa inserida, de forma aproximada, na escala equitemperada, fazendo uso da praticidade desta última nas mudanças de tonalidades de trechos musicais ou nas transposições. A Tabela 7 relaciona as notas e as respectivas frequências da oitava central da escala equitemperada, tendo como padrão a frequência de $440 \mathrm{~Hz}$ da nota Lá. Nesta escala, as alterações sustenido (\#) e bemol (b) definem notas enarmônicas, $d o_{\#}=r e_{b}, r e_{\#}=m i_{b}$, etc.

Tabela 7 - Intervalos em relação a Lá e as frequências (em Hertz) correspondentes às notas da oitava central na escala equitemperada. Apenas as notas sem alterações fazem parte da escala de dó maior.

\begin{tabular}{ccccccccccccc}
\hline $\mathrm{Do}$ & $\mathrm{Do}_{\#}$ & $\mathrm{Re}$ & $\mathrm{Re}_{\#}$ & $\mathrm{Mi}$ & $\mathrm{Fa}$ & $\mathrm{Fa}_{\#}$ & $\mathrm{Sol}$ & $\mathrm{Sol}_{\#}$ & $\mathrm{La}$ & $\mathrm{La} \#$ & $\mathrm{Si}$ & $\mathrm{Do}$ \\
$\mathrm{Do}$ & $\mathrm{Re}_{b}$ & $\mathrm{Re}$ & $\mathrm{Mi}_{b}$ & $\mathrm{Mi}$ & $\mathrm{Fa}$ & $\mathrm{Sol}_{b}$ & $\mathrm{Sol}$ & $\mathrm{La}_{b}$ & $\mathrm{La}$ & $\mathrm{Si}_{b}$ & $\mathrm{Si}$ & $\mathrm{Do}$ \\
\hline 1 & $r$ & $r^{2}$ & $r^{3}$ & $r^{4}$ & $r^{5}$ & $r^{6}$ & $r^{7}$ & $r^{8}$ & $r^{9}$ & $r^{10}$ & $r^{11}$ & 2 \\
262 & 277 & 294 & 311 & 330 & 349 & 370 & 392 & 415 & 440 & 466 & 494 & 523 \\
\hline
\end{tabular}

\section{Condições de consonância}

Os sons são ondas mecânicas que se propagam pelo ar, descritas por equações diferenciais lineares, tendo como fontes sistemas mecânicos vibrantes também descritos por equações diferenciais lineares. Deste modo os sons podem ser descritos como combinações lineares de ondas contendo os modos normais de vibração do sistema fonte. A principal propriedade do som é a altura, definida pela frequência mais baixa ou fundamental, as demais componentes harmônicas contribuindo para o timbre, uma das principais qualidades do som. Sons puros são os que tem uma única componente harmônica de frequência definida. Para entender as condições de consonância, considere dois sons puros de frequências diferentes $f_{1}$ e $f_{2}$ quando produzidos simultaneamente (harmonia) ou em sequência num curto intervalo de tempo (melodia). As observações do dia a dia sugerem que a qualidade do som resultante deve-se essencialmente às frequências dos sons primários, independente de suas amplitudes relativas ou eventuais diferenças de fases, entendendo-se por som primário o produzido por uma única fonte sonora.

Usando relações trigonométricas [5], as oscilações temporais, considerdas em fase, resultam [6]

$$
\begin{aligned}
& \cos 2 \pi f_{1} t+\cos 2 \pi f_{2} t= \\
& 2 \cos 2 \pi \frac{\left|f_{1}-f_{2}\right|}{2} t \cos 2 \pi \frac{\left(f_{1}+f_{2}\right)}{2} t,
\end{aligned}
$$

mostrando uma onda principal cuja frequência é a média das frequências dos sons primários,

$$
\bar{f}=f_{+}=\frac{\left(f_{1}+f_{2}\right)}{2}
$$

com amplitude modulada pela frequência de batimento

$$
f_{-}=\frac{\left(f_{2}-f_{1}\right)}{2} \text {. }
$$

Por considerações práticas e sem perda de generalidade, pode-se supor que $f_{2}>f_{1}$. Assim, pode-se ver que, se satisfeita a condição

$$
\left(f_{2}+f_{1}\right)=n\left(f_{2}-f_{1}\right),
$$

isto é, a relação de consonância

$$
\frac{f_{2}}{f_{1}}=\frac{(n+1)}{(n-1)},
$$

para $n$ inteiros maiores que $1(n>1)$, a onda resultante continuará periódica, comportando-se como uma combinação de dois sons harmônicos. Realmente, a relação trigonométrica (3) pode ser revertida para

$$
\begin{aligned}
& 2 \cos 2 \pi \frac{\left(f_{2}-f_{1}\right)}{2} t \cos 2 \pi \frac{\left(f_{1}+f_{2}\right)}{2} t= \\
& \cos 2 \pi(n-1) f_{0} t+\cos 2 \pi(n+1) f_{0} t,
\end{aligned}
$$

onde

$$
f_{0}=f_{2} /(n+1)=f_{1} /(n-1)
$$

assume o papel da frequência fundamental.

Embora a própria fundamental não esteja presente, as duas ondas são componentes harmônicas e portanto são consonantes se a consonância for interpretada como a ausência de distorções e quaisquer outras irregularidades na onda sonora como um todo. Em particular, $n=2$ e $n=3$ resultam nas relações $f_{2}=3 f_{1}$ e $f_{2}=2 f_{1}$, respectivamente, condições harmônicas trivialmente consonantes.

A relação de consonância, Eq. (7), é satisfeita quando a razão entre as frequências for um número racional e puder ser definido de tal forma que a diferença entre o numerador e o denominador seja igual a 2, pois

$$
(n+1)-(n-1)=2 .
$$

Por exemplo, o intervalo do - la,

$$
\frac{5}{3}=\frac{(4+1)}{(4-1)},
$$


o intervalo do - sol,

$$
\frac{3}{2}=\frac{6}{4}=\frac{(5+1)}{(5-1)},
$$

etc..

A Fig. 3 mostra as combinações de notas Dó e Lá no quadro (a), Dó e $M i$ no quadro (b) e Dó e $S o l$ no quadro (c), casos típicos de intervalos que satisfazem a relação de consonância, Eq. (7).
A Fig. 4 traz, nos quadros (a), (b) e (c) os padrões irregulares, com periodicidade não definida, que caracterizam as combinações dissonantes.

A Tabela 8 contém os intervalos entre todas as notas da escala de entonação justa mostrando que a maioria satisfaz a condição de consonância. O mesmo não ocorre na escala pitagórica, como mostra a Tabela 9, significando menos possibilidades para as construções de linhas melódicas ou de acordes.

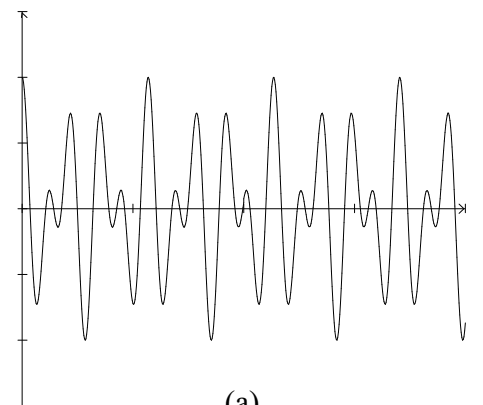

(a)

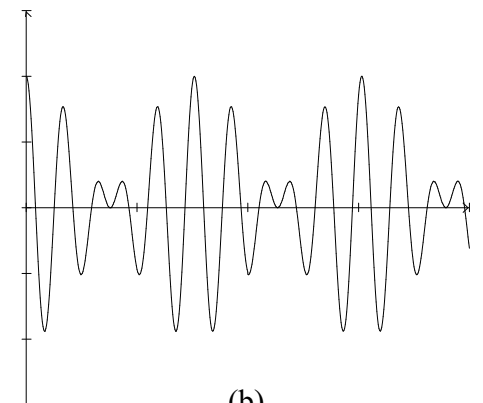

(b)

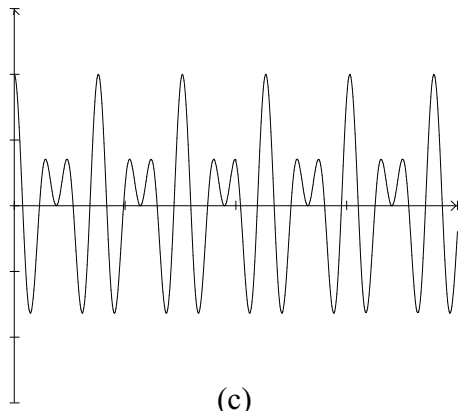

(c)

Figura 3 - Padrões regulares típicos de consonância, combinações de notas Dó e Lá em (a), Dó e Mi em (b) e Dó e Sol em (c).

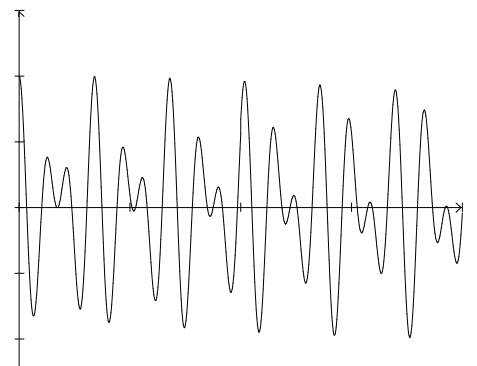

(a)

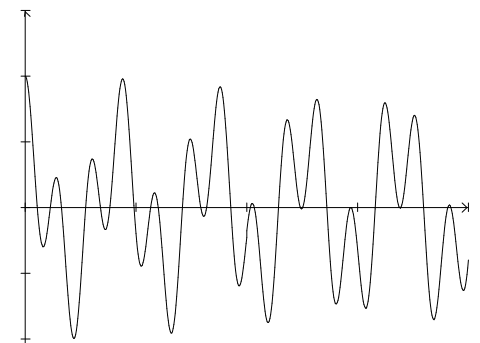

(b)

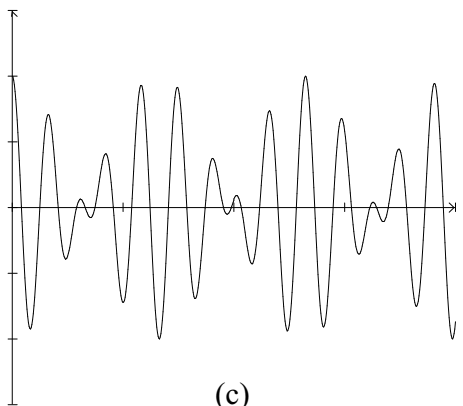

(c)

Figura 4 - Padrões irregulares não periódicos caracterizam a dissonância, combinação de Ré e Lá nos quadro (a) e combinações arbitrárias nos quadros (b) e (c).

Tabela 8 - Intervalos entre as notas, na escala de entonação justa.

\begin{tabular}{ccccccccc}
\hline & $d o$ & $r e$ & $m i$ & $f a$ & sol & $l a$ & si & $d o$ \\
\hline do & 1 & $9 / 8$ & $5 / 4$ & $4 / 3$ & $3 / 2$ & $5 / 3$ & $15 / 8$ & 2 \\
re & $8 / 9$ & 1 & $10 / 9$ & $32 / 27$ & $4 / 3$ & $40 / 27$ & $15 / 9$ & $16 / 9$ \\
$m i$ & $4 / 5$ & $9 / 10$ & 1 & $16 / 15$ & $6 / 5$ & $4 / 3$ & $3 / 2$ & $8 / 5$ \\
fa & $3 / 4$ & $27 / 32$ & $15 / 16$ & 1 & $9 / 8$ & $5 / 4$ & $45 / 32$ & $3 / 2$ \\
sol & $2 / 3$ & $4 / 3$ & $5 / 6$ & $8 / 9$ & 1 & $10 / 9$ & $5 / 4$ & $4 / 3$ \\
la & $3 / 5$ & $27 / 40$ & $3 / 4$ & $4 / 5$ & $9 / 10$ & 1 & $9 / 8$ & $6 / 5$ \\
si & $8 / 15$ & $9 / 15$ & $2 / 3$ & $32 / 45$ & $4 / 5$ & $8 / 9$ & 1 & $16 / 15$ \\
do & $1 / 2$ & $9 / 16$ & $5 / 8$ & $2 / 3$ & $3 / 4$ & $5 / 6$ & $15 / 16$ & 1 \\
\hline
\end{tabular}

Tabela 9 - Intervalos entre as notas, na escala pitagórica.

\begin{tabular}{ccccccccc}
\hline & $d o$ & $r e$ & $m i$ & $f a$ & sol & $l a$ & $s i$ & $d o$ \\
\hline$d o$ & 1 & $9 / 8$ & $81 / 64$ & $4 / 3$ & $3 / 2$ & $27 / 16$ & $243 / 128$ & 2 \\
$r e$ & $8 / 9$ & 1 & $9 / 8$ & $32 / 27$ & $4 / 3$ & $3 / 2$ & $27 / 16$ & $16 / 9$ \\
$m i$ & $64 / 81$ & $8 / 9$ & 1 & $256 / 243$ & $32 / 27$ & $4 / 3$ & $3 / 2$ & $512 / 81$ \\
fa & $3 / 4$ & $27 / 32$ & $243 / 256$ & 1 & $9 / 8$ & $81 / 64$ & $729 / 512$ & $3 / 2$ \\
sol & $2 / 3$ & $3 / 4$ & $27 / 32$ & $8 / 9$ & 1 & $9 / 8$ & $81 / 64$ & $4 / 3$ \\
la & $16 / 27$ & $2 / 3$ & $3 / 4$ & $64 / 81$ & $8 / 9$ & 1 & $9 / 8$ & $32 / 27$ \\
si & $128 / 243$ & $16 / 27$ & $2 / 3$ & $512 / 729$ & $64 / 81$ & $8 / 9$ & 1 & $256 / 243$ \\
do & $1 / 2$ & $9 / 16$ & $81 / 128$ & $2 / 3$ & $3 / 4$ & $27 / 32$ & $243 / 256$ & 1 \\
\hline
\end{tabular}


É de se esperar que quanto menor $n$ mais simples é a relação numérica (7) e melhor se manifeste a consonância. O intervalo de semitom 16/15 satisfaz a relação (7) para $n=31$ porém notas separadas por intervalo de semitom não são consideradas consonantes pelos músicos, em razão dos batimentos.

Veja que das Eqs. (4) e (5) resultam

$$
f_{-}=f_{0}
$$

e

$$
\bar{f}=f_{+}=n f_{0},
$$

garantindo um padrão regular de periodicidade para o som resultante. Quando as frequências dos sons primários são muito próximas, que implica $n$ muito grande e

$$
\bar{f}=n f_{0} \simeq f_{2} \simeq f_{1},
$$

o sistema auditivo não consegue discriminar os dois sons como distintos, percebendo-os como um único som com modulação na amplitude (batimento),

$$
\cos 2 \pi f_{1} t+\cos 2 \pi f_{2} t=\left(2 \cos 2 \pi f_{0} t\right) \cos 2 \pi n f_{0} t,
$$

a frequência de batimento $f_{-}=f_{0}$ abaixo do limiar de audição [7].

A Fig. 5 mostra a combinação das notas Dó e $S i$ no quadro (a), cujo intervalo que não satisfaz a condição de consonância (7), a combinação das notas $S i$ e $d o_{2}$ (intervalo de semitom) no quadro (b) que, embora satisfaça a condição (7) o efeito do batimento é dominante e, no quadro (c) a combinação da nota Dó com outro alterado de um coma, que resulta num batimento lento. O coma corresponde à "metade do semitom"e está próximo ao limiar da percepção humana. Se a frequência de batimento for muito pequeno em relação ao tempo envolvido, a modulação não será percebida e os dois sons serão confundidos como iguais. Este é o efeito prático que permite a escala de igual temperamento, os pequenos desvios nos intervalos entre as notas assim afinadas não sendo percebidos. Pela mesma razão, pequenos desvios da relação de consonância (7) não são percebidos.

\subsection{Amplitudes e fases relativas}

Nas análises acima, foram considerados sons puros de mesma amplitude e mesma fase, o que dificilmente poderiam ser reproduzidos na prática. Sendo assim, se amplitudes diferentes forem usadas, a Eq. (3) deve ser modificada para

$$
\begin{aligned}
& A_{1} \cos 2 \pi f_{1} t+A_{2} \cos 2 \pi f_{2} t= \\
& A_{1}\left[\cos 2 \pi f_{1} t+\cos 2 \pi f_{2} t\right]+\left(A_{2}-A_{1}\right) \cos 2 \pi f_{2} t,
\end{aligned}
$$

onde a Eq. (7) pode ser aplicada para os termos de igual amplitude. Então, usando a relação inversa (8), o termo à parte é reincorporada, resultando na expressão mais geral

$$
\begin{aligned}
& A_{1} \cos 2 \pi f_{1} t+A_{2} \cos 2 \pi f_{2} t= \\
& A_{1} \cos 2 \pi(n-1) f_{0} t+A_{2} \cos 2 \pi(n+1) f_{0} t,
\end{aligned}
$$

o que valida a relação de consonância (7) para condições mais gerais do mundo real, onde o controle fino da intensidade sonora nem sempre é possível.

A diferença de fase pode ser introduzida supondo que os sons primários sejam produzidos em instantes diferentes. Neste caso, no lado esquerdo da Eq. (13) pode ser reescrito como

$$
\begin{aligned}
& A_{1} \cos 2 \pi f_{1}\left(t-t_{1}\right)+A_{2} \cos 2 \pi f_{2}\left(t-t_{2}\right)= \\
& W[\cos ]+W[\operatorname{sen}],
\end{aligned}
$$

onde

$$
W[\cos ]=B_{1} \cos 2 \pi f_{1} t+B_{2} \cos 2 \pi f_{2} t
$$

e

$$
W[\operatorname{sen}]=C_{1} \operatorname{sen} 2 \pi f_{1} t+C_{2} \operatorname{sen} 2 \pi f_{2} t
$$

são funções auxiliares contendo termos em cosseno e seno, respectivamente, com coeficientes

$$
B_{1}=A_{1} \cos 2 \pi f_{1} t_{1}, B_{2}=A_{2} \cos 2 \pi f_{2} t_{2}
$$

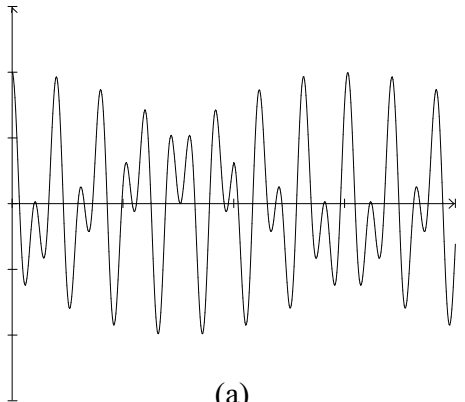

(a)

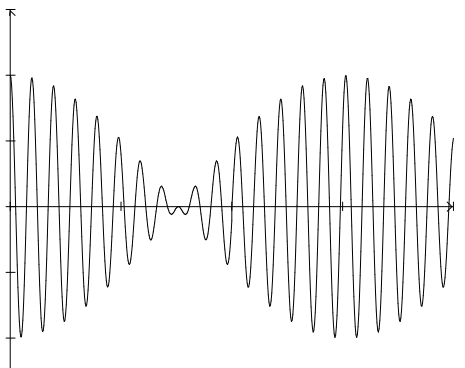

(b)

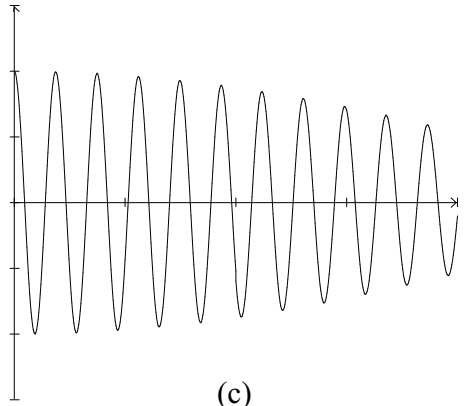

(c)

Figura 5 - Combinações de notas Dó e $S i$ em (a), $S i$ e $d o_{2}$ em (b) e Dó e som um coma acima em (c). 
e

$$
C_{1}=A_{1} \operatorname{sen} 2 \pi f_{1} t_{1}, C_{2}=A_{2} \operatorname{sen} 2 \pi f_{2} t_{2} .
$$

Supondo satisfeita a condição $(7)$, pode-se usar o desenvolvimento (13), resultando

$$
W[\cos ]=B_{1} \cos 2 \pi(n-1) f_{0} t+B_{2} \cos 2 \pi(n+1) f_{0} t .
$$

Para o termo em seno, usando relações trigonométricas

$$
\begin{aligned}
& \operatorname{sen} 2 \pi f_{1} t+\operatorname{sen} 2 \pi f_{2} t= \\
& 2 \cos 2 \pi \frac{\left(f_{2}-f_{1}\right)}{2} t \operatorname{sen} 2 \pi \frac{\left(f_{2}+f_{1}\right)}{2} t,
\end{aligned}
$$

o comportamento regular e periódico leva à mesma Eq. (7), resultando

$$
W[\operatorname{sen}]=C_{1} \operatorname{sen} 2 \pi(n-1) f_{0} t+C_{2} \operatorname{sen} 2 \pi(n+1) f_{0} t .
$$
geral

Revertendo os procedimentos, chega-se à expressão

$$
\begin{aligned}
& A_{1} \cos 2 \pi f_{1}\left(t-t_{1}\right)+A_{2} \cos 2 \pi f_{2}\left(t-t_{2}\right)= \\
& =A_{1} \cos 2 \pi(n-1) f_{0}\left(t-t_{1}\right)+ \\
& A_{2} \cos 2 \pi(n+1) f_{0}\left(t-t_{2}\right)
\end{aligned}
$$

garantindo que a relação de consonância aplica-se independentemente de amplitudes e fases relativas dos sons primários. A independência de fase é o que garante a melodia e a harmonia numa mesma escala musical.

\subsection{Combinações harmônicas}

Fontes sonoras são sistemas mecânicos postos em vibração, superposição linear de todos os modos normais de oscilação permitidos pelo sistema [4]. O som resultante contém exatamente os mesmos modos de oscilação da fonte, resultando num som característico que define a qualidade (timbre) do som, a altura definida pela frequência do modo fundamental.

Assim sendo, os sons, ao propagarem-se no meio, são combinações harmônicas contendo o modos normais defindos pelas fontes sonoras. Deste modo, a relação de consonância (7), além de independente das amplitudes e fases relativas dos sons primários, deve ser válido para as combinações harmônicas. Considerando os sons primários como combinações harmônicas cujas frequências fundamentais são $f_{1}$ e $f_{2}$,

$$
u_{1}(t)=\sum_{k=1}^{\infty} A_{k} \cos 2 \pi k f_{1} t
$$

e

$$
u_{2}(t)=\sum_{k=1}^{\infty} B_{k} \cos 2 \pi k f_{2} t
$$

resulta na composição (considerando por enquanto $\left.B_{k}=A_{k}\right)$

$$
\begin{aligned}
u(t) & =\sum_{k=1}^{\infty} A_{k}\left(\cos 2 \pi k f_{1} t+\cos 2 \pi k f_{2} t\right) \\
& =2 \sum_{k=1}^{\infty} A_{k} \cos 2 \pi k \frac{\left|f_{1}-f_{2}\right|}{2} t \cos 2 \pi k \frac{\left(f_{1}+f_{2}\right)}{2} t
\end{aligned}
$$

Aplicando a condição (7), supondo $f_{1}<f_{2}$, e revertendo a relação trigonométrica, Eqs. (8) e (13), chegase à forma geral

$u(t)=\sum_{k=1}^{\infty} A_{k} \cos 2 \pi k(n-1) f_{0} t+B_{k} \cos 2 \pi k(n+1) f_{0} t$

para a série harmônica, atestando que a relação de consonância (7) continua aplicável.

\section{Conclusões}

Embora consonância e dissonância sejam percepções com muita subjetividade, podendo variar entre indivíduos ou culturas, em termos gerais é de se esperar que a influência das condições físicas expressas através de relações matemáticas sejam fundamentais. Isto porque o som é uma manifestação física que pode ser descrita, tanto na produção como na propagação, através de leis físicas e equações matemáticas. Neste contexto é que se apresenta uma relação matemática exata para definir fisicamente as condições de consonância de ondas sonoras. Sob estas condições a onda resultante mantem a estrutura harmônica, tendo a frequência de batimento como a fundamental, embora a mesma não esteja presente, o que a diferencia das ondas primárias. A consonância é independente de fases e amplitudes relativas assim como das componentes harmônicas, e a relação de consonância proposta satisfaz a estas condições, dependendo apenas das frequências fundamentais das ondas primárias.

Quando a frequência de batimento é próximo ao limiar da audição, o efeito de modulação da amplitude torna-se mais importante, quebrando a percepção de consonância, mesmo que a condição física de consonância esteja satisfeita. Para ondas primárias com frequências muito próximas, o efeito de batimento é suave e praticamente imperceptível. A aplicação mais importante da consonância é na música, onde percebese que os intervalos entre as notas estruturadas numa escala musical são otimizadas para que satisfaçam mutuamente à condição de consonância. 


\section{Referências}

[1] Guilherme Schubert, Método Expositivo de Teoria Musical (Record, Rio de Janeiro, 1966).

[2] Harry F. Oslon, Music, Physics and Enginering (Dover, New York, 1967).

[3] Thomas D. Rossing, The Science of Sound (AddisonWesley, Reading, 1990).

[4] D. Halliday and R. Resnick, Physics (John Wiley \& Sons, New York, 1977). Edições em português são familiares aos estudantes de física; Richard P. Feynman, Robert B. Leighton and Mattew Sands, The Feynman Lectures on Physics (Addison-Wesley, Reading, 1963).
[5] Veja, por ex., Murray R. Spiegel, Manual de Fórmulas e Tabelas Matemáticas - (Coleção Schaum) (McGrawHill do Brasil, São Paulo, 1977).

[6] A.N. Tijonov and A. A. Samarsky, Equaciones de la Fisica Matematica (MIR, Moscow, 1972); George Arfken, Mathematical Methods for Physics (Academic Press, New York, 1970).

[7] Augustus L. Stanford, Foundations of Biophysics (Academic Press, New York, 1975); Arthur C. Guyton, Tratado de Fisiologia Médica (Ed. Guanabara Koogan, Rio de Janeiro, 1969). 